\title{
The Stochastic $\Theta$-Method for Nonlinear Stochastic Volterra Integro-Differential Equations
}

\author{
Peng $\mathrm{Hu}^{1}$ and Chengming Huang ${ }^{2}$ \\ ${ }^{1}$ School of Mathematics and Physics, China University of Geosciences, Wuhan 430074, China \\ ${ }^{2}$ School of Mathematics and Statistics, Huazhong University of Science and Technology, Wuhan 430074, China \\ Correspondence should be addressed to Peng Hu; hupeng1118@126.com
}

Received 6 May 2014; Accepted 28 August 2014; Published 27 October 2014

Academic Editor: Sanling Yuan

Copyright (c) 2014 P. Hu and C. Huang. This is an open access article distributed under the Creative Commons Attribution License, which permits unrestricted use, distribution, and reproduction in any medium, provided the original work is properly cited.

\begin{abstract}
The stochastic $\Theta$-method is extended to solve nonlinear stochastic Volterra integro-differential equations. The mean-square convergence and asymptotic stability of the method are studied. First, we prove that the stochastic $\Theta$-method is convergent of order $1 / 2$ in mean-square sense for such equations. Then, a sufficient condition for mean-square exponential stability of the true solution is given. Under this condition, it is shown that the stochastic $\Theta$-method is mean-square asymptotically stable for every stepsize if $1 / 2 \leq \theta \leq 1$ and when $0 \leq \theta<1 / 2$, the stochastic $\Theta$-method is mean-square asymptotically stable for some small stepsizes. Finally, we validate our conclusions by numerical experiments.
\end{abstract}

\section{Introduction}

In this paper we study the numerical solution of the $d$ dimensional nonlinear stochastic Volterra integro-differential equation (SVIDE) with convolution kernels

$$
\begin{aligned}
d x(t)= & f\left(x(t), \int_{0}^{t} G(t-s) x(s) d s\right) d t \\
& +g\left(x(t), \int_{0}^{t} H(t-s) x(s) d s\right) d W(t),
\end{aligned}
$$

with initial data $x(0)=x_{0}$. Here $W(t)$ is a scalar Brownian motion, and $f, g, G$, and $H$ are given functions which map

$$
\begin{aligned}
& f: R^{d} \times R^{d} \longrightarrow R^{d}, \quad g: R^{d} \times R^{d} \longrightarrow R^{d}, \\
& G: R \longrightarrow R^{d \times d}, \quad H: R \longrightarrow R^{d \times d} .
\end{aligned}
$$

In particular, (1) can be regarded as a stochastically perturbed problem of the deterministic nonlinear Volterra integro-differential equation (VIDE)

$$
x^{\prime}(t)=f\left(x(t), \int_{0}^{t} G(t-s) x(s) d s\right) .
$$

In the last decades, VIDEs have received a great deal of attention. A well-known example of this type is the Volterra population equation

$$
x^{\prime}(t)=a x(t)-b x^{2}(t)+x(t) \int_{0}^{t} G(t-s) x(s) d s
$$

For a general theory of VIDEs, we refer the reader to the classical book by Burton [1]. Also, many efficient numerical methods such as linear multistep methods, Runge-Kutta methods, and collocation methods have been constructed for VIDEs (see [2-4] and the extensive bibliography therein).

However, many real-world phenomena are subject to some random environmental effects. In recent years, the study of stability for SVIDEs has attracted the attention of many authors. For example, in 2000, Mao [5] studied the stability of the stochastic integro-differential equation as follows:

$$
d x(t)=f(x(t), t)+g\left(\int_{0}^{t} G(t-s) x(s) d s, t\right) d W(t),
$$

and several criteria on mean-square exponential stability and $L^{2}$-stability have been obtained. Later, Mao and Riedle [6] 
extended these results to a more general type of equation. Appleby [7] studied the almost sure asymptotic stability and integrability of the zero solution of a finite dimensional ItôVolterra equation.

Since the majority of stochastic differential equations cannot be solved analytically, numerical methods are adopted for obtaining approximate solutions. In recent years, a large number of numerical methods are constructed for stochastic ordinary differential equations (SODEs), such as stochastic linear multistep methods, stochastic Runge-Kutta methods, and stochastic Taylor methods. For more details, we refer the reader to the papers [8-11], the Ph.D. thesis by Burrage [12], and the book by Kloeden and Platen [13]. But for SVIDEs, up to now, there are only few results about the numerical approximation in the existing literatures. In [14, 15], Golec and Sathananthan studied the convergence of Euler-Maruyama scheme for SVIDEs under some different assumptions. And in [16], the analytical asymptotic stability and numerical asymptotic stability have been considered for a class of linear stochastic functional differential equation. The areas of the regions of asymptotic stability for some numerical methods are derived. However we have not found any stability results for numerical methods for SVIDEs (1).

Stochastic $\Theta$-method is a widely used method with strong convergence order $1 / 2$ for SODEs. If $\Theta=0$, it is the classical Euler-Maruyama method, and it is the Backward Euler method when $\Theta=1$. Similar to deterministic $A$ stability, Higham $[17,18]$ introduced stochastic $A$-stability which means that the problem stability implies the numerical method stability for any stepsize, and it is shown that the stochastic $\Theta$-method is stochastic $A$-stable if $1 / 2 \leq \theta \leq 1$.

In this paper, we apply the stochastic $\Theta$-method to SVIDE (1). The mean-square convergence and stability of the method are considered. It is proved that the stochastic $\Theta$-method for SVIDE has mean-square convergence order $1 / 2$. For the numerical stability analysis, first we extend the stability result in [5] to the case of SVIDE (1), and a sufficient condition for the mean-square exponential stability of the true solution is obtained. Under this condition, we then show that the stochastic $\Theta$-method is mean-square asymptotically stable for any stepsize if $1 / 2 \leq \theta \leq 1$, and when $0 \leq \theta<1 / 2$, the stochastic $\Theta$-method is mean-square asymptotically stable for some small stepsizes.

This paper is organized as follows. In Section 2, we study the mean-square convergence of the stochastic $\Theta$ method for SVIDEs. In Section 3, we investigate the meansquare stability of the method. In Section 4, some numerical experiments are given to validate our conclusions.

\section{Mean-Square Convergence of the Stochastic $\Theta$-Method}

Throughout this paper, we use the following notations. Let $\left(\Omega, \mathscr{F},\left\{\mathscr{F}_{t}\right\}_{t \geq 0}, P\right)$ be a complete probability space with a filtration $\left\{\mathscr{F}_{t}\right\}_{t \geq 0}$ satisfying normal conditions, namely, $\left\{\mathscr{F}_{t}\right\}_{t \geq 0}$ is increasing and right continuous with $\mathscr{F}_{0}$ containing all $P$ null sets. And let $W(t)$ be a scalar Brownian motion defined on the probability space. Let $\langle\cdot, \cdot\rangle$ be the Euclidean inner product in space $R^{d}$ and $|\cdot|$ be the corresponding norm. The matrix norm is denoted by $\|\cdot\|$, and $A^{T}$ denotes the transpose of a matrix $A$. $E(\cdot)$ stands for the mathematical expectation operator. For any vectors $x, y \in R^{d}$, we denote $\langle x, y\rangle=x \cdot y$.

For the mean-square convergence analysis, we consider the autonomous $d$-dimensional nonlinear SVIDE on $[0, T]$ where $T<\infty$

$$
\begin{aligned}
d x(t)= & f\left(x(t), \int_{0}^{t} G(t-s) x(s) d s\right) d t \\
& +g\left(x(t), \int_{0}^{t} H(t-s) x(s) d s\right) d W(t),
\end{aligned}
$$

with initial data $x(0)=x_{0}$. We assume that the functions $f$ and $g$ are globally Lipschitz continuous in all variables and satisfy linear growth condition, that is, there exist positive constants $L$ and $K$, such that for all $x, \bar{x}, y, \bar{y} \in R^{d}$,

$$
\begin{gathered}
|f(x, y)-f(\bar{x}, \bar{y})|^{2} \vee|g(x, y)-g(\bar{x}, \bar{y})|^{2} \\
\leq L\left(|x-\bar{x}|^{2}+|y-\bar{y}|^{2}\right) ; \\
|f(x, y)|^{2} \vee|g(x, y)|^{2} \leq K\left(1+|x|^{2}+|y|^{2}\right),
\end{gathered}
$$

where $a \vee b$ denotes the maximum of $a$ and $b$. Also, we assume that the functions $G$ and $H$ are continuous and satisfy

$$
\|G(t)-G(s)\| \vee\|H(t)-H(s)\| \leq \widehat{L}|t-s|,
$$

for all $t, s \in[0, T]$, which implies

$$
\|G(t)\| \vee\|H(t)\| \leq M<\infty,
$$

where $M$ and $\widehat{L}$ are positive constants. The above conditions (7)-(9) can guarantee that SVIDE (6) has a unique solution $x(t)$.

The following lemma will be used in our convergence analysis.

Lemma 1. Under conditions (8)-(9), the solution of SVIDE (6) has the property

$$
E\left(\sup _{0 \leq t \leq T}|x(t)|^{2}\right) \leq C_{1},
$$

with

$$
C_{1}:=\left(\frac{1}{2}+3 E\left|x_{0}\right|^{2}\right) e^{(6 T+24) K \bar{K} T},
$$

where

$$
\bar{K}=\max \left\{1, T^{2} M^{2}\right\} .
$$

Moreover, for any $0 \leq r<t \leq T$,

$$
E|x(t)-x(r)|^{2} \leq C_{2}(t-r),
$$

where $C_{2}=2 K \bar{K}(T+1)\left(1+2 C_{1}\right)$.

Proof. The proof of this lemma is similar to that of Lemma 5.5.2 in [19], and that of Theorem 2.3 in [20]. 
Now we apply the stochastic $\Theta$-method to SVIDE (6) and obtain the following numerical scheme:

$$
\begin{aligned}
& x_{n+1} \\
& =x_{n}+h\left[\theta f\left(x_{n+1}, z_{n+1}\right)+(1-\theta) f\left(x_{n}, z_{n}\right)\right] \\
& +g\left(x_{n}, \bar{z}_{n}\right) \Delta W_{n},
\end{aligned}
$$

where $h>0$ is a given stepsize, and $x_{n}$ is an approximation to $x\left(t_{n}\right)$ with $t_{n}=n h, n=1, \ldots, N=T / h$. The increment $\Delta W_{n}=W\left(t_{n+1}\right)-W\left(t_{n}\right)$ is an $N(0, h)$-distributed Gaussian random variable. And the arguments $z_{n}$ and $\bar{z}_{n}$ denote approximations to

$$
\int_{0}^{t_{n}} G\left(t_{n}-s\right) x(s) d s, \quad \int_{0}^{t_{n}} H\left(t_{n}-s\right) x(s) d s .
$$

In this paper, we choose the composite left rectangular formula to discretize the integral terms and obtain the following schemes

$$
z_{n}=h \sum_{j=0}^{n-1} G\left(t_{n}-t_{j}\right) x_{j}, \quad \bar{z}_{n}=h \sum_{j=0}^{n-1} H\left(t_{n}-t_{j}\right) x_{j} .
$$

Moreover we denote that

$$
\begin{aligned}
& z\left(t_{n}\right)=h \sum_{j=0}^{n-1} G\left(t_{n}-t_{j}\right) x\left(t_{j}\right), \\
& \bar{z}\left(t_{n}\right)=h \sum_{j=0}^{n-1} H\left(t_{n}-t_{j}\right) x\left(t_{j}\right) .
\end{aligned}
$$

In this section, without loss of generality, we assume $0<h<$ 1 .

Lemma 2. Under conditions (8)-(9), there exist positive constants $C_{3}, C_{4}$ such that

$$
\begin{aligned}
& E\left|\int_{0}^{t_{n}} G\left(t_{n}-s\right) x(s) d s-z\left(t_{n}\right)\right|^{2} \leq C_{3} h, \\
& E\left|\int_{0}^{t_{n}} H\left(t_{n}-s\right) x(s) d s-\bar{z}\left(t_{n}\right)\right|^{2} \leq C_{4} h .
\end{aligned}
$$

Proof. By (18), we have

$$
\begin{aligned}
& E\left|\int_{0}^{t_{n}} G\left(t_{n}-s\right) x(s) d s-z\left(t_{n}\right)\right|^{2} \\
& \quad=E\left|\int_{0}^{t_{n}} G\left(t_{n}-s\right) x(s) d s-h \sum_{j=0}^{n-1} G\left(t_{n}-t_{j}\right) x\left(t_{j}\right)\right|^{2} \\
& \quad=E\left|\sum_{j=0}^{n-1} \int_{t_{j}}^{t_{j+1}}\left[G\left(t_{n}-s\right) x(s)-G\left(t_{n}-t_{j}\right) x\left(t_{j}\right)\right] d s\right|^{2} \\
& \quad \leq n \sum_{j=0}^{n-1} E\left|\int_{t_{j}}^{t_{j+1}}\left[G\left(t_{n}-s\right) x(s)-G\left(t_{n}-t_{j}\right) x\left(t_{j}\right)\right] d s\right|^{2}
\end{aligned}
$$

$$
\begin{aligned}
\leq & 2 T \sum_{j=0}^{n-1} E \int_{t_{j}}^{t_{j+1}}\left\|G\left(t_{n}-s\right)-G\left(t_{n}-t_{j}\right)\right\|^{2}|x(s)|^{2} d s \\
& +2 T \sum_{j=0}^{n-1} E \int_{t_{j}}^{t_{j+1}}\left\|G\left(t_{n}-t_{j}\right)\right\|^{2}\left|x(s)-x\left(t_{j}\right)\right|^{2} d s .
\end{aligned}
$$

Therefore, by (9) and Lemma 1, we obtain

$$
\begin{gathered}
E\left|\int_{0}^{t_{n}} G\left(t_{n}-s\right) x(s) d s-z\left(t_{n}\right)\right|^{2} \\
\leq 2 T C_{1} \widehat{L} \sum_{j=0}^{n-1} \int_{t_{j}}^{t_{j+1}}\left(s-t_{j}\right)^{2} d s \\
\quad+2 T M^{2} C_{2} \sum_{j=0}^{n-1} \int_{t_{j}}^{t_{j+1}}\left(s-t_{j}\right) d s \\
\leq \frac{2 T^{2} C_{1} \widehat{L}}{3} h^{2}+T^{2} M^{2} C_{2} h .
\end{gathered}
$$

Since $0<h<1$, thus, there exists a constant $C_{3}$ which does not depend on $h$, such that (19) holds. Similar to the above proof, (20) also holds.

For the convergence analysis, the local truncation error is defined by

$$
\begin{aligned}
\delta_{n+1}=x\left(t_{n+1}\right) & \\
-\left\{x\left(t_{n}\right)+h[\right. & \theta f\left(x\left(t_{n+1}\right), z\left(t_{n+1}\right)\right) \\
& \left.+(1-\theta) f\left(x\left(t_{n}\right), z\left(t_{n}\right)\right)\right] \\
& \left.+g\left(x\left(t_{n}\right), \bar{z}\left(t_{n}\right)\right) \Delta W_{n}\right\},
\end{aligned}
$$

and the global error is defined by

$$
\epsilon_{n}=x\left(t_{n}\right)-x_{n} .
$$

It is obvious that $\epsilon_{n}$ is $\mathscr{F}_{t_{n}}$-measurable since both $x\left(t_{n}\right)$ and $x_{n}$ are $\mathscr{F}_{t_{n}}$-measurable random variables.

Definition 3. The stochastic $\Theta$-method (15)-(17) is said to be consistent with order $p_{1}$ in the mean and with order $p_{2}$ in the mean-square sense if the following estimations hold with $p_{2} \geq 1 / 2$ and $p_{1} \geq p_{2}+(1 / 2):$

$$
\begin{aligned}
& \max _{0 \leq n \leq N-1}\left(E\left(\left|E\left(\delta_{n+1} \mid \mathscr{F}_{t_{n}}\right)\right|^{2}\right)\right)^{1 / 2} \leq C h^{p_{1}}, \quad \text { as } h \longrightarrow 0 \\
& \max _{0 \leq n \leq N-1}\left(E\left|\delta_{n+1}\right|^{2}\right)^{1 / 2} \leq C h^{p_{2}}, \quad \text { as } h \longrightarrow 0
\end{aligned}
$$

where the constant $C$ does not depend on $h$ but may depend on $T$ and the initial data.

Lemma 4. Under conditions (7)-(9), the stochastic $\Theta$-method (15)-(17) is consistent with order $p_{1}=3 / 2$ in the mean and with order $p_{2}=1$ in the mean-square sense. 
Proof. Note that

$$
\begin{aligned}
& x\left(t_{n+1}\right)-x\left(t_{n}\right) \\
& =\int_{t_{n}}^{t_{n+1}} f\left(x(s), \int_{0}^{s} G(s-r) x(r) d r\right) d s \\
& \quad+\int_{t_{n}}^{t_{n+1}} g\left(x(s), \int_{0}^{s} H(s-r) x(r) d r\right) d W(s),
\end{aligned}
$$

then we have

$$
\begin{gathered}
E\left(\left|E\left(\delta_{n+1} \mid \mathscr{F}_{t_{n}}\right)\right|^{2}\right) \\
=E\left(\mid E\left(\int_{t_{n}}^{t_{n+1}} f\left(x(s), \int_{0}^{s} G(s-r) x(r) d r\right) d s\right.\right. \\
-h\left(\theta f\left(x\left(t_{n+1}\right), z\left(t_{n+1}\right)\right)\right. \\
\left.\left.\left.+(1-\theta) f\left(x\left(t_{n}\right), z\left(t_{n}\right)\right)\right) \mid \mathscr{F}_{t_{n}}\right)\left.\right|^{2}\right) \\
\leq E\left(\mid \int_{t_{n}}^{t_{n+1}} f\left(x(s), \int_{0}^{s} G(s-r) x(r) d r\right) d s\right. \\
-h\left(\theta f\left(x\left(t_{n+1}\right), z\left(t_{n+1}\right)\right)\right. \\
\left.\left.\left.+(1-\theta) f\left(x\left(t_{n}\right), z\left(t_{n}\right)\right)\right)\left.\right|^{2} \mid \mathscr{F}_{t_{n}}\right)\right) \\
=E\left(\mid \int_{t_{n}}^{t_{n+1}} f\left(x(s), \int_{0}^{s} G(s-r) x(r) d r\right) d s\right. \\
-h\left(\theta f\left(x\left(t_{n+1}\right), z\left(t_{n+1}\right)\right)\right. \\
\left.\left.+(1-\theta) f\left(x\left(t_{n}\right), z\left(t_{n}\right)\right)\right)\left.\right|^{2}\right)
\end{gathered}
$$$$
\leq 2 I_{1}+2 I_{2}
$$

where

$$
\begin{aligned}
& I_{1} \\
& =E\left(\mid \int_{t_{n}}^{t_{n}+(1-\theta) h}\left(f\left(x(s), \int_{0}^{s} G(s-r) x(r) d r\right)\right.\right. \\
& \left.\left.-f\left(x\left(t_{n}\right), z\left(t_{n}\right)\right)\right)\left.d s\right|^{2}\right) \\
& =E\left(\mid \int_{t_{n}+(1-\theta) h}^{t_{n+1}}\left(f\left(x\left(t_{n+1}\right), z\left(t_{n+1}\right)\right)\right.\right. \\
& \left.\left.-f\left(x(s), \int_{0}^{s} G(s-r) x(r) d r\right)\right)\left.d s\right|^{2}\right) .
\end{aligned}
$$

By Hölder inequality and condition (7), we obtain

$$
\begin{aligned}
I_{1} \leq & (1-\theta) h \int_{t_{n}}^{t_{n}+(1-\theta) h} E \mid f\left(x(s), \int_{0}^{s} G(s-r) x(r) d r\right) \\
& -\left.f\left(x\left(t_{n}\right), z\left(t_{n}\right)\right)\right|^{2} d s \\
\leq & (1-\theta) h L \\
& \times \int_{t_{n}}^{t_{n}+(1-\theta) h}\left(E\left|x(s)-x\left(t_{n}\right)\right|^{2}\right. \\
& \left.+E\left|\int_{0}^{s} G(s-r) x(r) d r-z\left(t_{n}\right)\right|^{2}\right) d s .
\end{aligned}
$$

By (9)-(11) and Lemma 2, we get

$$
\begin{aligned}
& E\left|\int_{0}^{s} G(s-r) x(r) d r-z\left(t_{n}\right)\right|^{2} \\
& \leq 2 E \mid \int_{0}^{s}(G(s-r)) x(r) d r \\
& \quad-\left.\int_{0}^{t_{n}}\left(G\left(t_{n}-r\right)\right) x(r) d r\right|^{2} \\
& \quad+2 E\left|\int_{0}^{t_{n}}\left(G\left(t_{n}-r\right)\right) x(r) d r-z\left(t_{n}\right)\right|^{2} \\
& \leq 4 E\left|\int_{0}^{t_{n}}\left(G(s-r)-G\left(t_{n}-r\right)\right) x(r) d r\right|^{2} \\
& \quad+4 E\left|\int_{t_{n}}^{s}(G(s-r)) x(r) d r\right|^{2}+2 C_{3} h \\
& \leq 4 T^{2} C_{1} \widehat{L}^{2}\left(s-t_{n}\right)^{2}+4 C_{1} M^{2}\left(s-t_{n}\right)^{2}+2 C_{3} h .
\end{aligned}
$$

Therefore, by (14), we have

$I_{1} \leq(1-\theta) h L$

$$
\begin{aligned}
& \times \int_{t_{n}}^{t_{n}+(1-\theta) h}\left(C_{2}\left(s-t_{n}\right)\right. \\
& \left.\quad+4 C_{1}\left(T^{2} \widehat{L}^{2}+M^{2}\right)\left(s-t_{n}\right)^{2}+2 C_{3} h\right) d s \\
& =\left(\frac{1}{2} C_{2}(1-\theta)^{3}+2 C_{3}(1-\theta)^{2}\right) L h^{3} \\
& +\frac{4 C_{1}\left(T^{2} \widehat{L}^{2}+M^{2}\right) L}{3}(1-\theta)^{4} h^{4} .
\end{aligned}
$$

Since $0<h<1$, there exists a constant $C_{5}$ which does not depend on $h$, such that

$$
I_{1} \leq C_{5} h^{3}
$$


We can also prove that there exists a constant $C_{6}$ which does not depend on $h$, such that

$$
I_{2} \leq C_{6} h^{3}
$$

then we have

$$
E\left(\left|E\left(\delta_{n+1} \mid \mathscr{F}_{t_{n}}\right)\right|^{2}\right)^{1 / 2} \leq \sqrt{2\left(C_{5}+C_{6}\right)} h^{3 / 2} .
$$

Now we prove that the stochastic $\Theta$-method is consistent with order 1 in the mean-square sense. Using the inequality $(a+b+c)^{2} \leq 3 a^{2}+3 b^{2}+3 c^{2}$, Hölder's inequality, and Doob's martingale inequality, we have

$$
\begin{aligned}
& E\left(\left|\delta_{n+1}\right|^{2}\right) \\
& \leq 3(1-\theta) h \\
& \quad \times \int_{t_{n}}^{t_{n}+(1-\theta) h} E \mid f\left(x(s), \int_{0}^{s} G(s-r) x(r) d r\right) \\
& \quad-\left.f\left(x\left(t_{n}\right), z\left(t_{n}\right)\right)\right|^{2} d s \\
& \quad+3 \theta h \int_{t_{n}+(1-\theta) h}^{t_{n+1}} \mid f\left(x\left(t_{n+1}\right), z\left(t_{n+1}\right)\right) \\
& \quad+3 \int_{t_{n}}^{t_{n+1}} E \mid g\left(x(s), \int_{0}^{s} H(s-r) x(r) d r\right) \\
& \triangleq 3(1-\theta) h \widetilde{I}_{1}+3 \theta h \widetilde{I}_{2}+3 \widetilde{I}_{3} .
\end{aligned}
$$

By (7), (14), and (30), we get

$$
\begin{aligned}
& h \widetilde{I}_{1} \\
& \leq L h \int_{t_{n}}^{t_{n}+(1-\theta) h}\left(E\left|x(s)-x\left(t_{n}\right)\right|^{2}\right. \\
& \left.+E\left|\int_{0}^{s} G(s-r) x(r) d r-z\left(t_{n}\right)\right|^{2}\right) d s \\
& \leq L h \int_{t_{n}}^{t_{n}+(1-\theta) h}\left(C_{2}\left(s-t_{n}\right)+4 T^{2} C_{1}^{2} \widehat{L}^{2}\left(s-t_{n}\right)^{2}\right. \\
& \left.+4 C_{1}^{2} M^{2}\left(s-t_{n}\right)^{2}+2 C_{3}^{2} h^{2}\right) d s \\
& =\frac{1}{2} L C_{2}(1-\theta)^{3} h^{3}+C_{7} h^{4},
\end{aligned}
$$

where $C_{7}$ is independent of $h$. Similarly, we can obtain

$$
h \widetilde{I}_{2} \leq C_{8} h^{3}+C_{9} h^{4}, \quad \widetilde{I}_{3} \leq C_{10} h^{2}+C_{11} h^{3},
$$

where $C_{8}-C_{11}$ are independent of $h$. Since $0<h<1$, we obtain

$$
E\left(\left|\delta_{n+1}\right|^{2}\right) \leq C_{12} h^{2}
$$

where $C_{12}$ depends on $C_{2}, C_{7}-C_{11}$. The proof of the lemma is completed.

Definition 5. The stochastic $\Theta$-method (15)-(17) is said to be convergent in mean-square sense with order $p$, if

$$
\max _{0 \leq n \leq N}\left(E\left|\epsilon_{n}\right|^{2}\right)^{1 / 2} \leq C h^{p} \quad \text { as } h \longrightarrow 0
$$

Theorem 6. Under conditions (7)-(9), the numerical solution produced by the stochastic $\Theta$-method (15)-(17) converges to the exact solution of (6) with order $1 / 2$ in the mean-square sense.

Proof. Using the definition of the global error, we have

$$
\begin{array}{rl}
\epsilon_{n+1} & x\left(t_{n+1}\right)-x_{n+1} \\
= & x\left(t_{n+1}\right)-x\left(t_{n}\right) \\
& -h\left[\theta f\left(x\left(t_{n+1}\right), z\left(t_{n+1}\right)\right)+(1-\theta) f\left(x\left(t_{n}\right), z\left(t_{n}\right)\right)\right] \\
& -g\left(x\left(t_{n}\right), \bar{z}\left(t_{n}\right)\right) \Delta W_{n}+x\left(t_{n}\right) \\
& +h\left[\theta f\left(x\left(t_{n+1}\right), z\left(t_{n+1}\right)\right)+(1-\theta) f\left(x\left(t_{n}\right), z\left(t_{n}\right)\right)\right] \\
& +g\left(x\left(t_{n}\right), \bar{z}\left(t_{n}\right)\right) \Delta W_{n}-x_{n} \\
& -h\left[\theta f\left(x_{n+1}, z_{n+1}\right)+(1-\theta) f\left(x_{n}, z_{n}\right)\right] \\
& -g\left(x_{n}, \bar{z}_{n}\right) \Delta W_{n} \\
= & \delta_{n+1}+\epsilon_{n}+u_{n},
\end{array}
$$

where

$$
\begin{aligned}
u_{n}=h[ & \theta f\left(x\left(t_{n+1}\right), z\left(t_{n+1}\right)\right) \\
& \left.+(1-\theta) f\left(x\left(t_{n}\right), z\left(t_{n}\right)\right)\right] \\
+ & g\left(x\left(t_{n}\right), \bar{z}\left(t_{n}\right)\right) \Delta W_{n} \\
& -h\left[\theta f\left(x_{n+1}, z_{n+1}\right)+(1-\theta) f\left(x_{n}, z_{n}\right)\right] \\
& -g\left(x_{n}, \bar{z}_{n}\right) \Delta W_{n} .
\end{aligned}
$$

Squaring both sides of (40), taking expectation and absolute values, yields

$$
\begin{aligned}
E\left(\left|\epsilon_{n+1}\right|^{2}\right) & \\
\leq & 2 E\left(\left|\delta_{n+1}\right|^{2}\right)+E\left(\left|\epsilon_{n}\right|^{2}\right)+2 E\left(\left|u_{n}\right|^{2}\right) \\
& +2\left|E\left(\epsilon_{n} \cdot \delta_{n+1}\right)\right|+2\left|E\left(\epsilon_{n} \cdot u_{n}\right)\right| .
\end{aligned}
$$


We now estimate the separate terms in (42) individually and in sequence. Due to the consistency condition in the meansquare sense of the method, we have

$$
E\left(\left|\delta_{n+1}\right|^{2}\right) \leq \widetilde{C}_{1} h^{2}
$$

By using the inequality $(a+b+c)^{2} \leq 3 a^{2}+3 b^{2}+3 c^{2}$ and condition (7), we obtain

$$
\begin{aligned}
E\left(\left|u_{n}\right|^{2}\right) \leq & 3 h^{2} \theta^{2} L E\left(\left|\epsilon_{n+1}\right|^{2}\right) \\
& +3 h^{2} \theta^{2} L E\left(\left|z\left(t_{n+1}\right)-z_{n+1}\right|^{2}\right) \\
& +3 h^{2}(1-\theta)^{2} L E\left(\left|\epsilon_{n}\right|^{2}\right) \\
& +3 h^{2}(1-\theta)^{2} L E\left(\left|z\left(t_{n}\right)-z_{n}\right|^{2}\right) \\
& +3 h L E\left(\left|\epsilon_{n}\right|^{2}\right)+3 h L E\left(\left|\bar{z}\left(t_{n}\right)-\bar{z}_{n}\right|^{2}\right) .
\end{aligned}
$$

Note that

$$
\begin{aligned}
& E\left(\left|z\left(t_{n+1}\right)-z_{n+1}\right|^{2}\right) \\
& =E\left(\left|h \sum_{j=0}^{n} G\left(t_{n+1}-t_{j}\right) \epsilon_{j}\right|^{2}\right) \\
& \quad \leq h^{2} \sum_{j=0}^{n}\left|G\left(t_{n+1}-t_{j}\right)\right| \cdot \sum_{j=0}^{n}\left|G\left(t_{n+1}-t_{j}\right)\right| E\left(\left|\epsilon_{j}\right|^{2}\right) \\
& \quad \leq h M^{2} T \sum_{j=0}^{n} E\left(\left|\epsilon_{j}\right|^{2}\right), \\
& E\left(\left|\bar{z}\left(t_{n}\right)-\bar{z}_{n}\right|^{2}\right) \leq h M^{2} T \sum_{j=0}^{n-1} E\left(\left|\epsilon_{j}\right|^{2}\right),
\end{aligned}
$$

then we have

$$
\begin{aligned}
E\left(\left|u_{n}\right|^{2}\right) \leq & 3 h^{2} \theta^{2} L E\left(\left|\epsilon_{n+1}\right|^{2}\right) \\
& +3 h^{3} \theta^{2} L M^{2} T \\
& \times \sum_{j=0}^{n} E\left(\left|\epsilon_{j}\right|^{2}\right)+3 h^{2}(1-\theta)^{2} L E\left(\left|\epsilon_{n}\right|^{2}\right) \\
& +3 h^{3}(1-\theta)^{2} L M^{2} T \sum_{j=0}^{n-1} E\left(\left|\epsilon_{j}\right|^{2}\right) \\
& +3 h L E\left(\left|\epsilon_{n}\right|^{2}\right)+3 h^{2} L M^{2} T \sum_{j=0}^{n-1} E\left(\left|\epsilon_{j}\right|^{2}\right) .
\end{aligned}
$$

Due to properties of conditional expectation and the consistency condition in the mean sense,

$$
\begin{aligned}
2 \mid E & \left(\epsilon_{n} \cdot \delta_{n+1}\right) \mid \\
& =2\left|E\left(\epsilon_{n} \cdot E\left(\delta_{n+1} \mid \mathscr{F}_{t_{n}}\right)\right)\right| \\
& \leq 2\left(E\left(\left|\epsilon_{n}\right|^{2}\right)\right)^{1 / 2} \cdot\left(E\left(\left|E\left(\delta_{n+1} \mid \mathscr{F}_{t_{n}}\right)\right|^{2}\right)\right)^{1 / 2} \\
& \leq \widetilde{C}_{2} h^{2}+h E\left(\left|\epsilon_{n}\right|^{2}\right) .
\end{aligned}
$$

Using the Lipschtiz condition (7) and properties of conditional expectation, we obtain

$$
\begin{aligned}
2 \mid E & \left(\epsilon_{n} \cdot u_{n}\right) \mid \\
& \leq 2 E\left(\left|\epsilon_{n}\right| \cdot\left|E\left(u_{n} \mid \mathscr{F}_{t_{n}}\right)\right|\right) \\
& \leq 2 h \theta E\left(\left|\epsilon_{n}\right| \cdot \mid E\left(f\left(x\left(t_{n+1}\right), z\left(t_{n+1}\right)\right)\right.\right.
\end{aligned}
$$

$$
\left.\left.-f\left(x_{n+1}, z_{n+1}\right) \mid \mathscr{F}_{t_{n}}\right) \mid\right)
$$

$$
\begin{aligned}
& +2 h(1-\theta) E \\
& \times\left(\left|\epsilon_{n}\right| \cdot\left|f\left(x\left(t_{n}\right), z\left(t_{n}\right)\right)-f\left(x_{n}, z_{n}\right)\right|\right) \\
\leq & h E\left(\left|\epsilon_{n}\right|^{2}\right)+\theta \operatorname{LhE}\left(\left|\epsilon_{n+1}\right|^{2}\right) \\
& +\theta L h E\left(\left|z\left(t_{n+1}\right)-z_{n+1}\right|^{2}\right) \\
& +(1-\theta) \operatorname{LhE}\left(\left|\epsilon_{n}\right|^{2}\right) \\
& +(1-\theta) \operatorname{LhE}\left(\left|z\left(t_{n}\right)-z_{n}\right|^{2}\right) \\
\leq & h E\left(\left|\epsilon_{n}\right|^{2}\right)+\theta L h E\left(\left|\epsilon_{n+1}\right|^{2}\right) \\
& +\theta L h^{2} M^{2} T \sum_{j=0}^{n} E\left(\left|\epsilon_{j}\right|^{2}\right) \\
& +(1-\theta) \operatorname{LhE}^{2}\left(\left|\epsilon_{n}\right|^{2}\right) \\
& +(1-\theta) \operatorname{Lh}^{2} M^{2} T \sum_{j=0}^{n-1} E\left(\left|\epsilon_{j}\right|^{2}\right) .
\end{aligned}
$$

Substituting (43) and (46)-(48) into (42), we have

$$
\begin{aligned}
(1- & \left.6 h^{2} \theta^{2} L-h \theta L\right) E\left(\left|\epsilon_{n+1}\right|^{2}\right) \\
\leq & E\left(\left|\epsilon_{n}\right|^{2}\right)+\widetilde{C}_{3} h E\left(\left|\epsilon_{n}\right|^{2}\right)+\widetilde{C}_{4} h^{2} \sum_{j=0}^{n} E\left(\left|\epsilon_{j}\right|^{2}\right) \\
& +\widetilde{C}_{5} h^{3} \sum_{j=0}^{n} E\left(\left|\epsilon_{j}\right|^{2}\right)+\left(2 \widetilde{C}_{1}+\widetilde{C}_{2}\right) h^{2},
\end{aligned}
$$


where

$$
\begin{aligned}
& \widetilde{C}_{3}=2+6 L+(1-\theta) L, \\
& \widetilde{C}_{4}=6(1-\theta)^{2} L+7 L M^{2} T, \\
& \widetilde{C}_{5}=6 \theta^{2} L M^{2} T+6(1-\theta)^{2} L M^{2} T .
\end{aligned}
$$

Since $0 \leq \theta \leq 1$, for sufficiently small stepsize $h$, there exists a constant $\kappa \in(0,1)$, such that

$$
\begin{gathered}
1-6 h^{2} \theta^{2} L-h \theta L \geq 1-2 h \theta L \geq \kappa, \\
\widetilde{C}_{4} h^{2}+\widetilde{C}_{5} h^{3} \leq 2 \widetilde{C}_{4} h^{2} .
\end{gathered}
$$

Let

$$
R_{n}=\max _{1 \leq i \leq n}\left(E\left(\left|\epsilon_{i}\right|^{2}\right)\right)
$$

then we have

$$
\begin{aligned}
R_{n+1} & \leq \frac{1+\widetilde{C}_{3} h}{1-2 h \theta L} R_{n}+\frac{2 \widetilde{C}_{4} T h}{1-2 h \theta L} R_{n}+\frac{2 \widetilde{C}_{1}+\widetilde{C}_{2}}{1-2 h \theta L} h^{2} \\
& \leq\left[1+\frac{2 \theta L+\widetilde{C}_{3}+2 \widetilde{C}_{4} T}{\kappa} h\right] R_{n}+\frac{2 \widetilde{C}_{1}+\widetilde{C}_{2}}{\kappa} h^{2} .
\end{aligned}
$$

Denote

$$
\widehat{C}=\frac{2 \theta L+\widetilde{C}_{3}+2 \widetilde{C}_{4} T}{\kappa},
$$

and note that $R_{0}=0$, thus we obtain

$$
\begin{aligned}
R_{n+1} & \leq(1+\widehat{C} h) R_{n}+\frac{2 \widetilde{C}_{1}+\widetilde{C}_{2}}{\kappa} h^{2} \\
& \leq(1+\widehat{C} h)^{n+1} R_{0}+\frac{2 \widetilde{C}_{1}+\widetilde{C}_{2}}{\kappa} h^{2} \sum_{j=0}^{n}(1+\widehat{C} h)^{j} \\
& =\frac{2 \widetilde{C}_{1}+\widetilde{C}_{2}}{\widehat{C}}\left((1+\widehat{C} h)^{n+1}-1\right) h \\
& \leq \frac{2 \widetilde{C}_{1}+\widetilde{C}_{2}}{\widehat{C} \kappa}\left(e^{\hat{C} h(n+1)}-1\right) h \leq \frac{2 \widetilde{C}_{1}+\widetilde{C}_{2}}{\widehat{C} \kappa}\left(e^{\widehat{C} T}-1\right) h .
\end{aligned}
$$

From the above, the theorem is proved.

\section{Stability Analysis}

In this section, we will discuss the analytical stability and numerical stability of SVIDE (1). First, the analytical stability is derived by the technique in [5]. We extend the exponential stability result to a more general type. And then the meansquare asymptotic stability of the stochastic $\Theta$-method is investigated.

Let us give the definition of the mean-square exponential stability (see Mao [19]).
Definition 7. The solution $x(t)$ of SVIDE (1) is said to be mean-square exponentially stable if there exists a pair of positive constants $\lambda$ and $\widetilde{C}$ such that

$$
E|x(t)|^{2} \leq \widetilde{C} E\left|x_{0}\right|^{2} e^{-\lambda t} \quad \text { on } t \geq 0
$$

for all initial data $x_{0} \in R^{d}$.

Definition 8. The solution $x(t)$ of SVIDE (1) is said to be mean-square asymptotically stable if

$$
\lim _{t \rightarrow \infty} E|x(t)|^{2}=0,
$$

for all initial data $x_{0} \in R^{d}$.

Obviously, (56) implies (57).

Theorem 9. Assume that there exist six positive constants $\lambda_{1}$, $\lambda_{2}, \lambda_{3}, \lambda_{4}, \beta$, and $\gamma$ such that

$$
\begin{gathered}
2 x^{T} f(x, 0) \leq-\lambda_{1}|x|^{2}, \\
|f(x, y)-f(x, 0)| \leq \lambda_{2}|y|, \\
|g(x, y)|^{2} \leq \lambda_{3}|x|^{2}+\lambda_{4}|y|^{2},
\end{gathered}
$$

for all $x, y \in R^{d}$. And the convolution kernel functions $G$ and $H$ decay exponentially fast, that is

$$
\|G(t)\| \vee\|H(t)\| \leq \beta e^{-\gamma t}, \quad t \geq 0 .
$$

If

$$
-\lambda_{1}+\lambda_{2}+\lambda_{3}+\left(\lambda_{2}+\lambda_{4}\right) \frac{\beta^{2}}{\gamma^{2}}<0
$$

then for any given initial data $x_{0}$, there exists a pair of positive constants $\rho$ and $C$ such that the solution $x(t)$ of SVIDE (1) has property that

$$
E|x(t)|^{2} \leq C E\left|x_{0}\right|^{2} e^{-\rho t}, \quad \forall t \geq 0,
$$

which implies (57).

Proof. The proof of this theorem is similar to that of Theorem 2.1 in [5] by choosing the Lyapunov function $V(t, x)=e^{\lambda_{1} t}|x|^{2}$.

Remark 10. If we apply Theorem 9 to problem (5), then the corresponding mean-square stability condition is

$$
\lambda_{4} \beta^{2}<\lambda_{1} \gamma^{2} .
$$

This condition is in accordance with the assumption of Theorem 2.1 in Mao's papaer [5].

In the following, we study the mean-square asymptotic stability of the stochastic $\Theta$-method for SVIDE (1). 
Definition 11. The numerical solution $\left\{x_{n}\right\}_{n \geq 0}$ to SVIDE (1) is said to be mean-square asymptotically stable if the numerical solution has the property that

$$
\lim _{n \rightarrow \infty} E\left|x_{n}\right|^{2}=0
$$

for any initial data $x_{0}$.

Theorem 12. Under conditions (58)-(62), the stochastic $\Theta$ method is mean-square asymptotically stable for any stepsize $h>0$, if $1 / 2 \leq \theta \leq 1$.

Proof. By (15), we have

$$
F\left(x_{n+1}\right)=F\left(x_{n}\right)+h f\left(x_{n}, z_{n}\right)+g\left(x_{n}, \bar{z}_{n}\right) \Delta W_{n},
$$

where

$$
F\left(x_{n}\right)=x_{n}-\theta h f\left(x_{n}, z_{n}\right) .
$$

Consequently

$$
\begin{aligned}
\left|F\left(x_{n+1}\right)\right|^{2} \\
=\left|F\left(x_{n}\right)\right|^{2}+(1-2 \theta) h^{2}\left|f\left(x_{n}, z_{n}\right)\right|^{2} \\
\quad+\left|g\left(x_{n}, \bar{z}_{n}\right) \Delta W_{n}\right|^{2}+2\left\langle x_{n}, h f\left(x_{n}, z_{n}\right)\right\rangle \\
\quad+2\left\langle F\left(x_{n}\right)+h f\left(x_{n}, z_{n}\right), g\left(x_{n}, \bar{z}_{n}\right) \Delta W_{n}\right\rangle .
\end{aligned}
$$

Taking expectation on both sides of (68) and by $1 / 2 \leq \theta \leq 1$, it follows that

$$
\begin{aligned}
E\left|F\left(x_{n+1}\right)\right|^{2} \leq & E\left|F\left(x_{n}\right)\right|^{2}+h E\left|g\left(x_{n}, \bar{z}_{n}\right)\right|^{2} \\
& +2 E\left\langle x_{n}, h f\left(x_{n}, z_{n}\right)\right\rangle .
\end{aligned}
$$

By conditions (58)-(60), we have

$$
\begin{aligned}
E\left|F\left(x_{n+1}\right)\right|^{2} & \\
\leq & E\left|F\left(x_{n}\right)\right|^{2}+h\left(-\lambda_{1}+\lambda_{2}+\lambda_{3}\right) E\left|x_{n}\right|^{2} \\
& +h \lambda_{2} E\left|z_{n}\right|^{2}+h \lambda_{4} E\left|\bar{z}_{n}\right|^{2} .
\end{aligned}
$$

And by (61) and the Jensen inequality, we derive that

$$
\begin{aligned}
\left|z_{n}\right|^{2} & =\left|h \sum_{j=0}^{n-1} G\left(t_{n}-t_{j}\right) x_{j}\right|^{2} \leq \beta^{2}\left(h \sum_{j=0}^{n-1} e^{-\gamma\left(t_{n}-t_{j}\right)}\left|x_{j}\right|\right)^{2} \\
& \leq \beta^{2}\left(h \sum_{j=0}^{n-1} e^{-\gamma\left(t_{n}-t_{j}\right)}\right) \cdot\left(h \sum_{j=0}^{n-1} e^{-\gamma\left(t_{n}-t_{j}\right)}\left|x_{j}\right|^{2}\right) \\
& \leq \frac{\beta^{2}}{\gamma}\left(h \sum_{j=0}^{n-1} e^{-\gamma\left(t_{n}-t_{j}\right)}\left|x_{j}\right|^{2}\right), \\
& \left|\bar{z}_{n}\right|^{2} \leq \frac{\beta^{2}}{\gamma}\left(h \sum_{j=0}^{n-1} e^{-\gamma\left(t_{n}-t_{j}\right)}\left|x_{j}\right|^{2}\right) .
\end{aligned}
$$

Substituting (71) into (70), we have

$$
\begin{aligned}
E\left|F\left(x_{n+1}\right)\right|^{2} & \\
\leq & E\left|F\left(x_{n}\right)\right|^{2}+h\left(-\lambda_{1}+\lambda_{2}+\lambda_{3}\right) E\left|x_{n}\right|^{2} \\
& +h\left(\lambda_{2}+\lambda_{4}\right) \frac{\beta^{2}}{\gamma}\left(h \sum_{j=0}^{n-1} e^{-\gamma\left(t_{n}-t_{j}\right)} E\left|x_{j}\right|^{2}\right),
\end{aligned}
$$

which implies

$$
\begin{aligned}
E\left|F\left(x_{n+1}\right)\right|^{2} & \leq\left|F\left(x_{0}\right)\right|^{2}+h\left(-\lambda_{1}+\lambda_{2}+\lambda_{3}\right) \sum_{i=0}^{n} E\left|x_{i}\right|^{2} \\
& +h\left(\lambda_{2}+\lambda_{4}\right) \frac{\beta^{2}}{\gamma} \sum_{i=0}^{n}\left(h \sum_{j=0}^{i-1} e^{-\gamma\left(t_{i}-t_{j}\right)} E\left|x_{j}\right|^{2}\right) \\
\leq & E\left|F\left(x_{0}\right)\right|^{2}+h\left(-\lambda_{1}+\lambda_{2}+\lambda_{3}\right) \sum_{i=0}^{n} E\left|x_{i}\right|^{2} \\
& +h\left(\lambda_{2}+\lambda_{4}\right) \frac{\beta^{2}}{\gamma} \sum_{j=0}^{n-1}\left(h \sum_{i=j+1}^{n} e^{-\gamma\left(t_{i}-t_{j}\right)} E\left|x_{j}\right|^{2}\right) \\
\leq & E\left|F\left(x_{0}\right)\right|^{2}+h\left(-\lambda_{1}+\lambda_{2}+\lambda_{3}\right) \\
& \times \sum_{i=0}^{n} E\left|x_{i}\right|^{2}+h\left(\lambda_{2}+\lambda_{4}\right) \frac{\beta^{2}}{\gamma^{2}} \sum_{j=0}^{n-1} E\left|x_{j}\right|^{2} \\
\leq & E\left|F\left(x_{0}\right)\right|^{2}+h\left(-\lambda_{1}+\lambda_{2}+\lambda_{3}+\left(\lambda_{2}+\lambda_{4}\right) \frac{\beta^{2}}{\gamma^{2}}\right) \\
& \times \sum_{i=0}^{n} E\left|x_{i}\right|^{2} \cdot
\end{aligned}
$$

Therefore by condition (62), we have

$$
\sum_{i=0}^{n} E\left|x_{i}\right|^{2} \leq C E\left|F\left(x_{0}\right)\right|^{2}
$$

for any $n>0$, where $C=-1 / h\left(-\lambda_{1}+\lambda_{2}+\lambda_{3}+\left(\lambda_{2}+\right.\right.$ $\left.\left.\lambda_{4}\right)\left(\beta^{2} / \gamma^{2}\right)\right)$. Let $n \rightarrow \infty$, we find that the infinite series $\sum_{i=0}^{\infty} E\left|x_{i}\right|^{2}$ is bounded, then we have

$$
\lim _{n \rightarrow \infty} E\left|x_{n}\right|^{2}=0
$$

which shows that the stochastic $\Theta$-method is mean-square asymptotically stable. The proof of the theorem is completed.

Theorem 13. Assume that conditions (58)-(62) hold and that function $f$ satisfies the linear growth condition; namely, there exists a constant $\widehat{K}>0$ such that

$$
|f(x, y)|^{2} \leq \widehat{K}\left(|x|^{2}+|y|^{2}\right) .
$$


If $0 \leq \theta<1 / 2$, then the stochastic $\Theta$-method is mean-square asymptotically stable when the stepsize $h \in\left(0, h_{0}\right)$, where

$$
h_{0}=\frac{\lambda_{1}-\lambda_{2}-\lambda_{3}-\left(\lambda_{2}+\lambda_{4}\right)\left(\beta^{2} / \gamma^{2}\right)}{(1-2 \theta) \widehat{K}\left(1+\left(\beta^{2} / \gamma^{2}\right)\right)} .
$$

Proof. By (68) and the linear growth condition (76), we have

$$
\begin{aligned}
& E\left|F\left(x_{n+1}\right)\right|^{2} \\
& \leq E\left|F\left(x_{n}\right)\right|^{2} \\
&+\left[h\left(-\lambda_{1}+\lambda_{2}+\lambda_{3}\right)+(1-2 \theta) h^{2} \widehat{K}\right] E\left|x_{n}\right|^{2} \\
&+\left[h \lambda_{2}+(1-2 \theta) h^{2} \widehat{K}\right] E\left|z_{n}\right|^{2}+h \lambda_{4} E\left|\bar{z}_{n}\right|^{2}
\end{aligned}
$$

which implies

$$
\begin{aligned}
& E\left|F\left(x_{n+1}\right)\right|^{2} \\
& \leq E\left|F\left(x_{0}\right)\right|^{2} \\
& +\left[h\left(-\lambda_{1}+\lambda_{2}+\lambda_{3}\right)+(1-2 \theta) h^{2} \widehat{K}\right] \sum_{i=0}^{n} E\left|x_{i}\right|^{2} \\
& +\left[h \lambda_{2}+(1-2 \theta) h^{2} \widehat{K}\right] \frac{\beta^{2}}{\gamma^{2}} \sum_{j=0}^{n-1} E\left|x_{j}\right|^{2} \\
& +h \lambda_{4} \frac{\beta^{2}}{\gamma^{2}} \sum_{j=0}^{n-1} E\left|x_{j}\right|^{2} \\
& \leq E\left|F\left(x_{0}\right)\right|^{2}+\left[h\left(-\lambda_{1}+\lambda_{2}+\lambda_{3}+\left(\lambda_{2}+\lambda_{4}\right) \frac{\beta^{2}}{\gamma^{2}}\right)\right.
\end{aligned}
$$

By condition (62), $0 \leq \theta<1 / 2$ and $h \in\left(0, h_{0}\right)$, we receive

$$
\begin{array}{r}
h\left(-\lambda_{1}+\lambda_{2}+\lambda_{3}+\left(\lambda_{2}+\lambda_{4}\right) \frac{\beta^{2}}{\gamma^{2}}\right) \\
+h^{2}(1-2 \theta) \widehat{K}\left(1+\frac{\beta^{2}}{\gamma^{2}}\right)<0 .
\end{array}
$$

Therefore we conclude that

$$
\lim _{n \rightarrow \infty} E\left|x_{n}\right|^{2}=0
$$

The proof of the theorem is completed.

Remark 14. In recent years, there are so many valuable results on the stability analysis of numerical methods for stochastic delay integro-differential equations. However, these results can not be applied to stochastic Volterra integro-differential equations directly since they have essential differences. Moreover, for the deterministic cases, these two issues are studied separately in the past few decades; please refer to the book [2] by Brunner.

\section{Numerical Experiments}

In this section, some numerical examples are given to validate our conclusions. First we illustrate the theoretical convergence order of stochastic $\Theta$-method. Here we consider the following linear SVIDE:

$$
\begin{aligned}
& d x(t) \\
& =\left(a x(t)+b \int_{0}^{t} e^{-3(t-s)} x(s) d s\right) d t \\
& \quad+\left(c x(t)+d \int_{0}^{t} e^{-2(t-s)} x(s) d s\right) d W(t), \\
& t \in[0, T],
\end{aligned}
$$

with the initial data $x(0)=1$. And we choose the parameters $a=-1, b=0.5, c=0.5, d=0.5$, and $T=1$. Since the true solution of (82) can not be obtained, thus we compute a reference solution with the trapezoidal rule $(\theta=0.5)$ under a very small stepsize $h=2^{-15}$. The mean-square error is denoted as follows:

$$
\begin{aligned}
\operatorname{err} & :=\max _{1 \leq n \leq N}\left(\frac{1}{M} \sum_{j=1}^{M}\left|x\left(t_{n}, \omega_{j}\right)-x_{n}\left(\omega_{j}\right)\right|^{2}\right)^{1 / 2} \\
& \approx \max _{1 \leq n \leq N}\left(E\left|\varepsilon_{n}\right|^{2}\right)^{1 / 2},
\end{aligned}
$$

where $N$ denotes the number of steps and $M$ is the number of sample paths. In our experiments, we choose $M=500$. Here we choose four different numerical methods with $\theta=0$ (classical Euler-Maruyama method), $\theta=0.3, \theta=0.7$, and $\theta=1$ (Backward Euler method), and the numerical results are presented in Table 1. From the table, one can easily receive that the mean-square convergence order of the stochastic $\Theta$ method for SVIDEs is $1 / 2$. Also, we illustrate the convergence result by Figure 1, where the reference dotted curve has a slope $1 / 2$.

In the rest of this section, some numerical experiments are given to validate our stability results. Consider the following nonlinear SVIDE:

$$
\begin{aligned}
& d x(t) \\
& =\left(a x(t)+b \sin \left(\int_{0}^{t} e^{-3(t-s)} x(s) d s\right)\right) d t \\
& \quad+\left(c x(t)+d \sin \left(\int_{0}^{t} e^{-4(t-s)} x(s) d s\right)\right) d W(t), \\
& t \geq 0,
\end{aligned}
$$

with the initial data $x(0)=1$. We choose the parameters $a=$ $-10, b=3, c=2$, and $d=1$. By conditions (58)-(61), we have

$$
\begin{aligned}
& \lambda_{1}=20, \quad \lambda_{2}=3, \quad \lambda_{3}=6, \quad \lambda_{4}=3, \\
& \widehat{K}=130, \quad \beta=1, \quad \gamma=3 \text {. }
\end{aligned}
$$


TABLE 1: Mean-square errors of the four methods for (82).

\begin{tabular}{lcccc}
\hline & $\theta=0$ & $\theta=0.3$ & $\theta=0.7$ & $\theta=1$ \\
\hline$h=2^{-4}$ & 0.03366846 & 0.03079402 & 0.02981565 & 0.03126486 \\
$h=2^{-5}$ & 0.02200355 & 0.02111309 & 0.02098438 & 0.02167162 \\
$h=2^{-6}$ & 0.01594512 & 0.01560370 & 0.01551390 & 0.01572048 \\
$h=2^{-7}$ & 0.01064680 & 0.01058695 & 0.01064387 & 0.01078690 \\
$h=2^{-8}$ & 0.00726183 & 0.00724734 & 0.00727678 & 0.00733495 \\
\hline
\end{tabular}

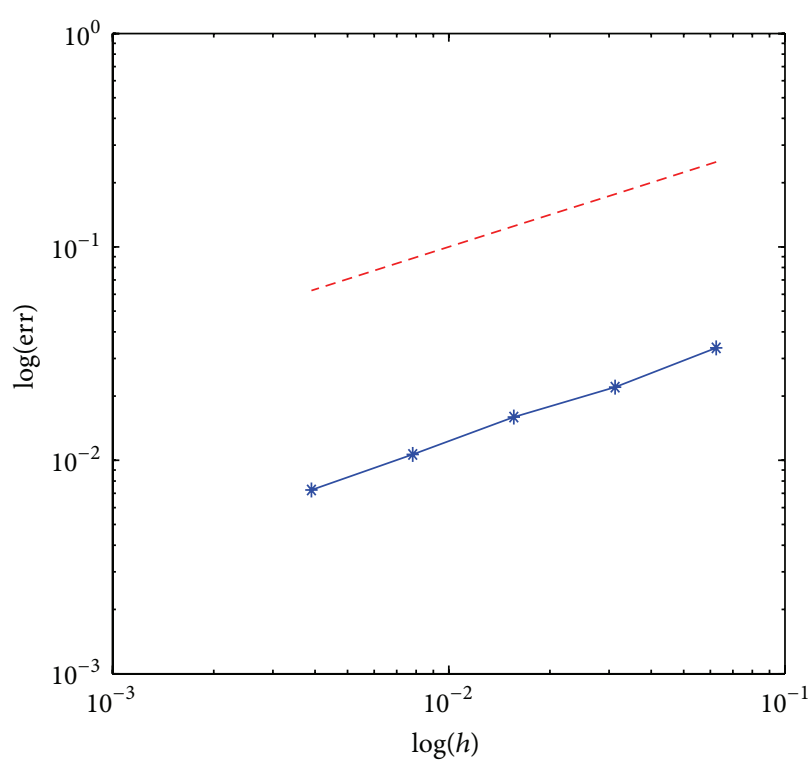

$\rightarrow-\theta=0$

(a)

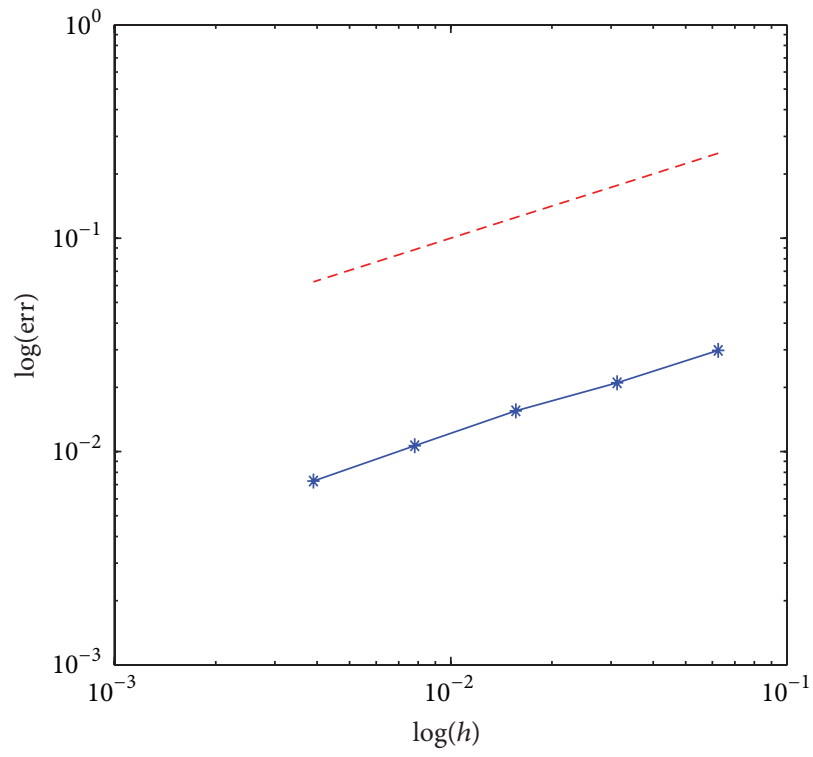

$-\theta=0.7$

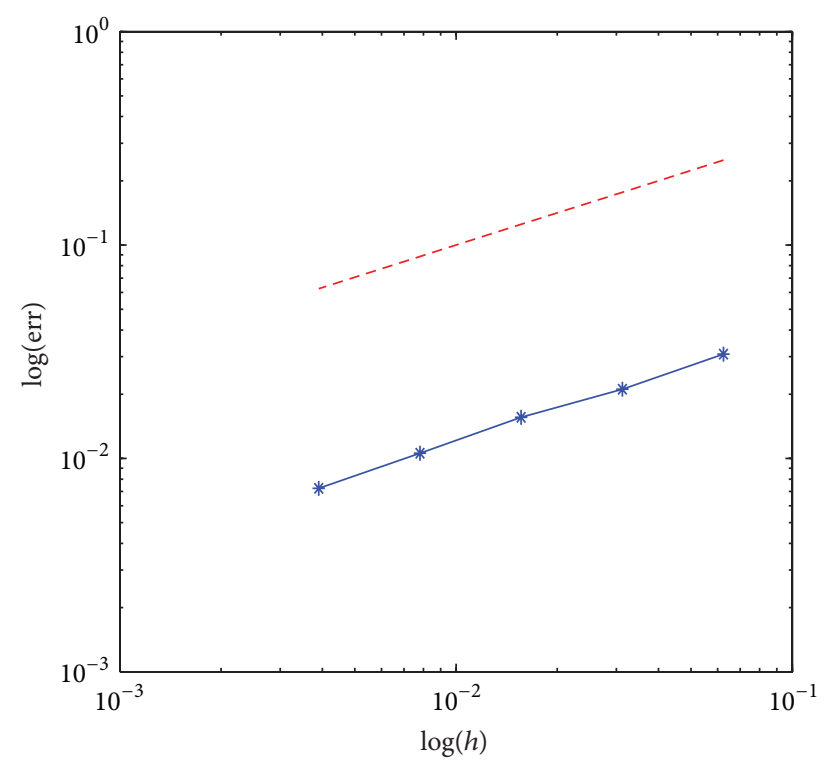

$\rightarrow-\theta=0.3$

(b)

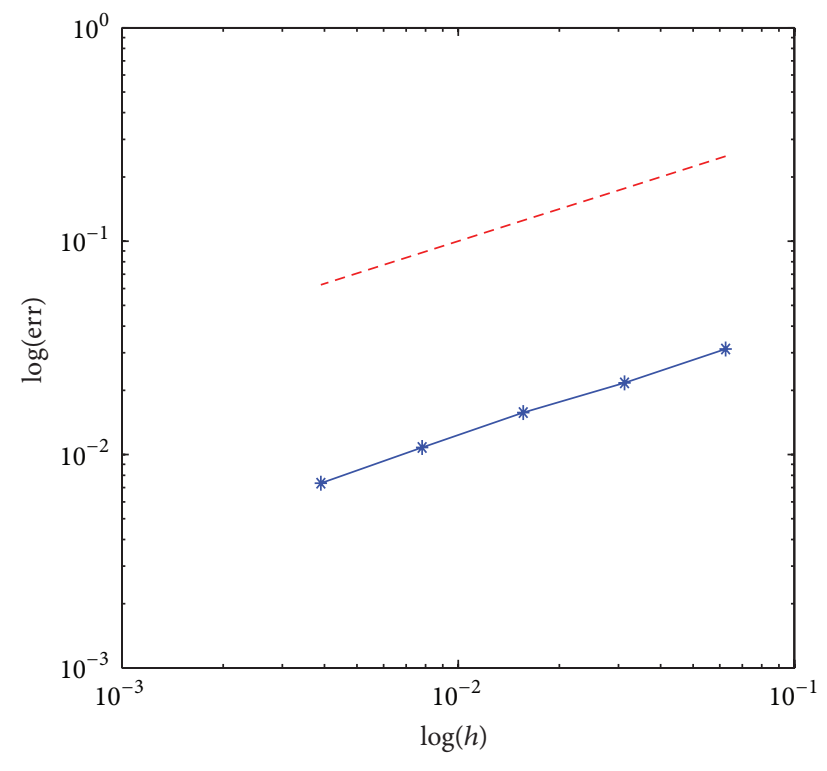

$\rightarrow-\theta=1$

(d)

(c)

FIGURE 1: Mean-square error plots of the four methods for (82). 


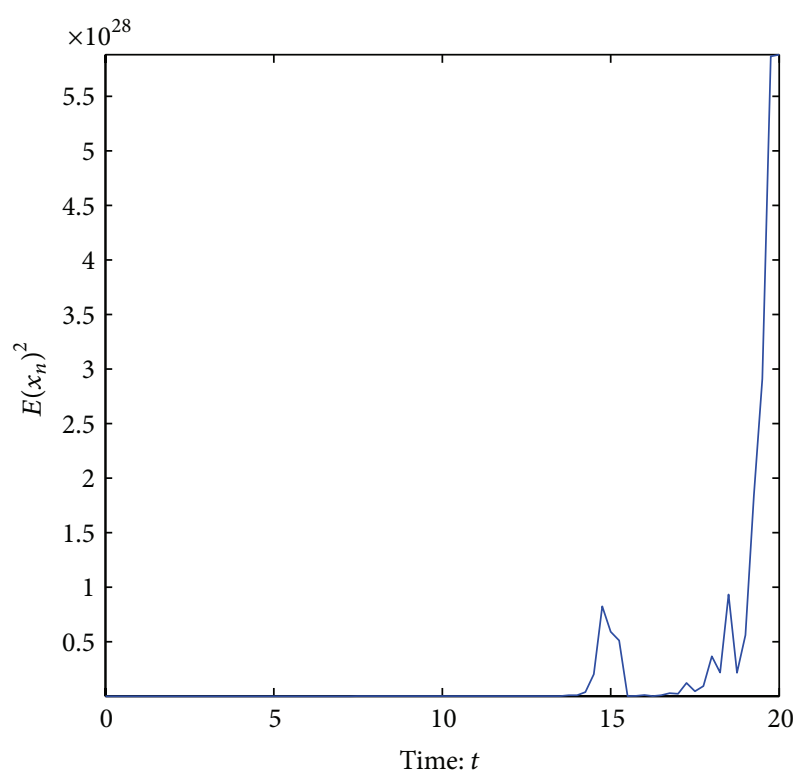

$-\theta=0, h=1 / 4$

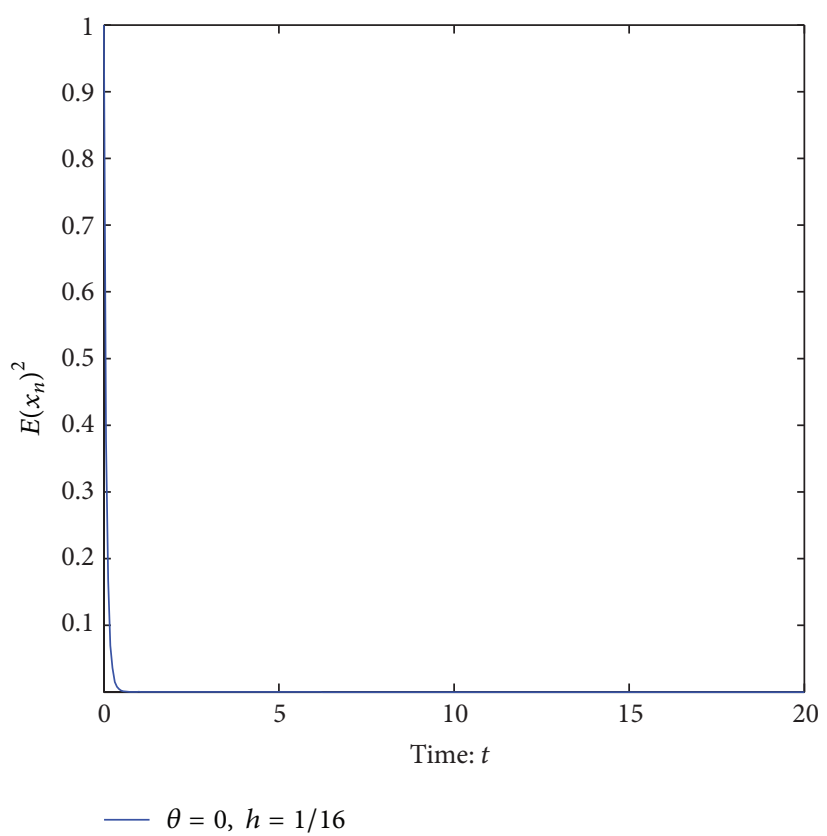

(b)

Figure 2: Numerical simulation with $\theta=0$ under different stepsizes $h=1 / 4$ and $h=1 / 16$.

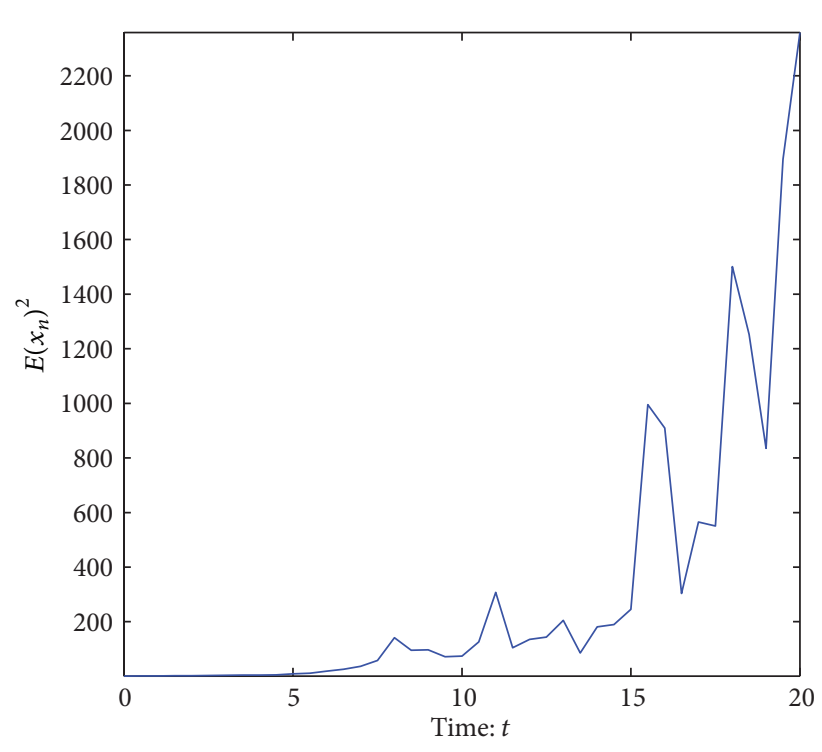

$-\theta=0.3, h=1 / 2$

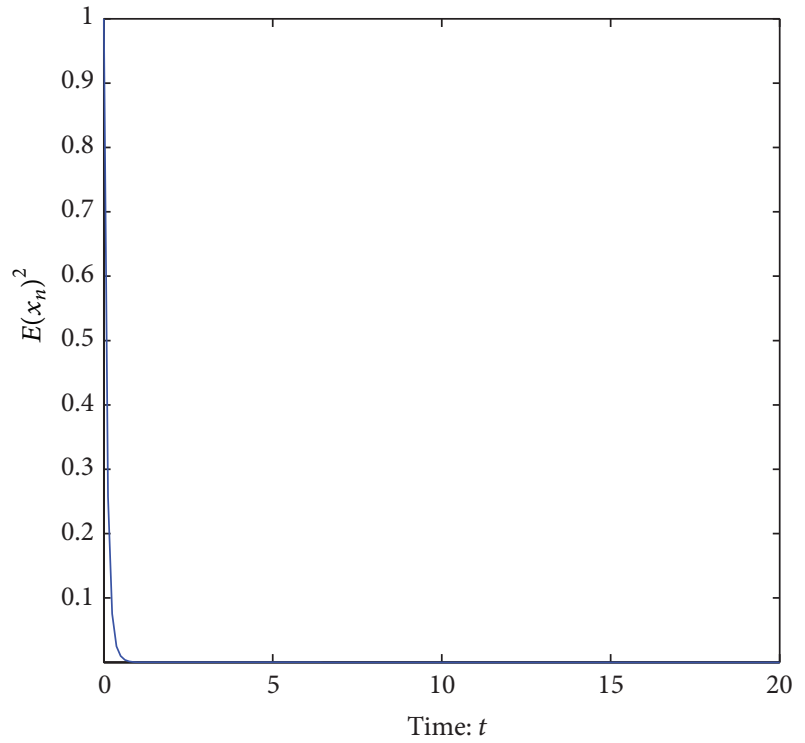

$-\theta=0.3, h=1 / 8$

FIGURE 3: Numerical simulation with $\theta=0.3$ under different stepsizes $h=1 / 2$ and $h=1 / 8$.

Therefore,

$$
-\lambda_{1}+\lambda_{2}+\lambda_{3}+\left(\lambda_{2}+\lambda_{4}\right) \frac{\beta^{2}}{\gamma^{2}}=-\frac{31}{3}<0,
$$

which implies that the true solution of (84) is mean-square asymptotically stable.

In the following numerical experiments, we will show the numerical mean-square asymptotic stability of the stochastic
$\Theta$-method with different parameters. Here we choose $\theta=0$, $\theta=0.3, \theta=0.8$, and $\theta=1$ where $0,0.3 \in[0,1 / 2)$ and $0.8,1 \epsilon$ $[1 / 2,1]$. By Theorem 13, we have

(i) if $\theta=0, h_{0}=93 / 1300$. When the stepsize $h \epsilon$ $(0,93 / 1300)$, the Euler-Maruyama method is meansquare asymptotically stable; 


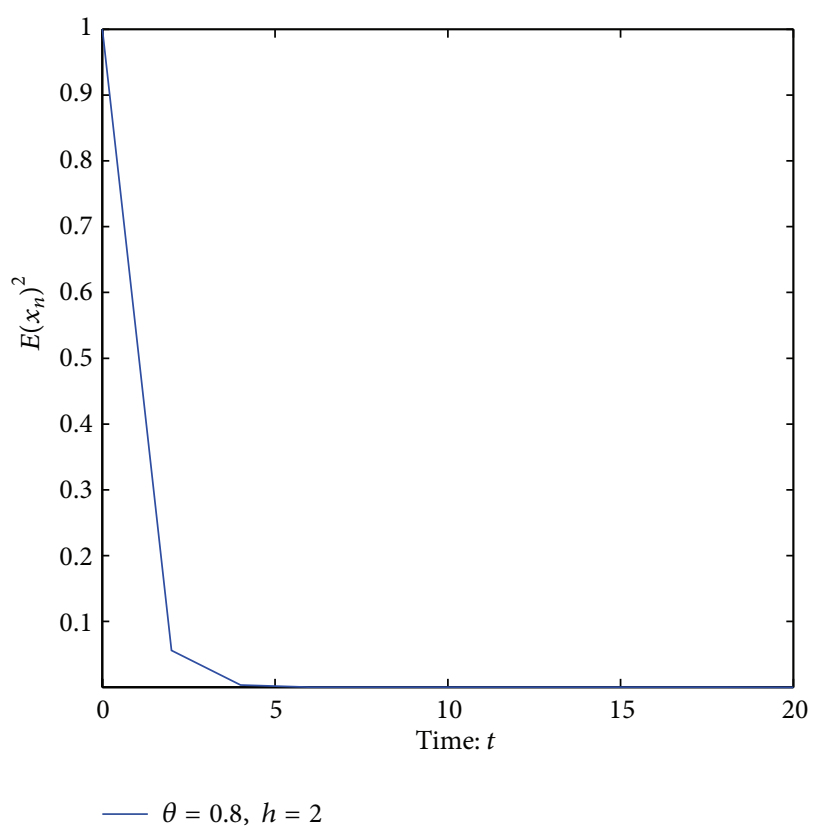

(a)

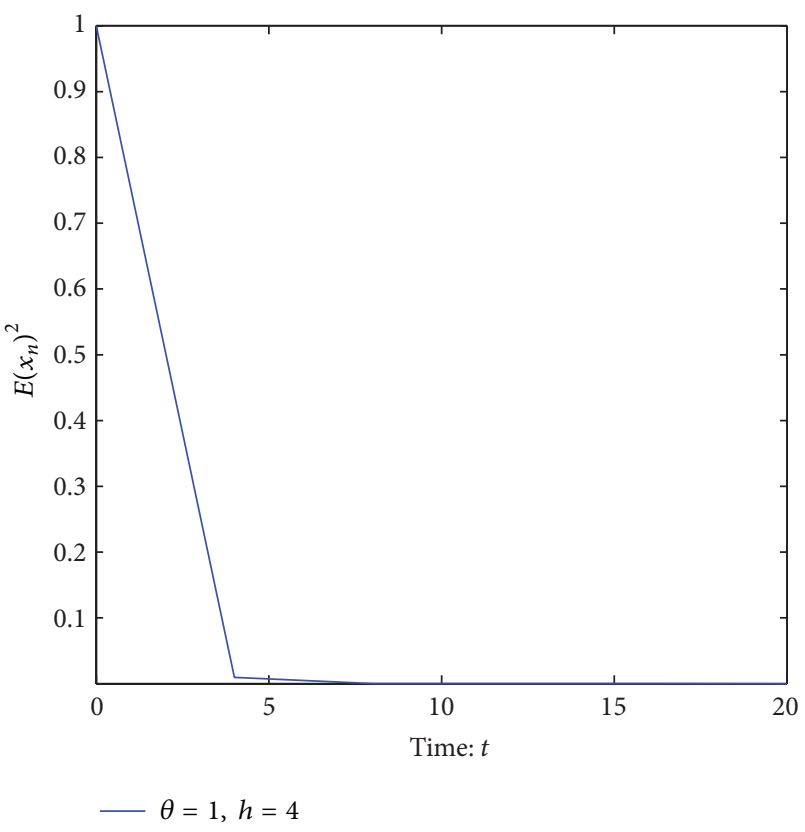

(b)

Figure 4: Numerical simulation with $\theta=0.8$ and $\theta=1$ under even large stepsizes $h=2$ and $h=3$.

(ii) if $\theta=0.3, h_{0}=93 / 520$. When the stepsize $h \in$ $(0,93 / 520)$, the method is mean-square asymptotically stable.

In Figures 2 and 3, one can find that when the stepsize $h \in$ $\left(0, h_{0}\right), E\left|x_{n}\right|^{2}$ tends to zero, but it tends to infinity when the stepsize is even large. Since the stability conditions are sufficient, one may find that the restriction of the stepsize in Theorem 13 is not optimal.

Theorem 12 says that when $\theta \in[1 / 2,1]$, the stochastic $\Theta$ method is mean-square asymptotically stable for any stepsize, and the following numerical experiments demonstrate this for a selection stepsize (see Figure 4).

\section{Conclusions}

In this paper, we first studied the mean-square convergence the stochastic $\Theta$-method for stochastic Volterra integrodifferential equations and proved that the stochastic $\Theta$ method has mean-square convergence order $1 / 2$ when the functions $f$ and $g$ in (1) are globally Lipschitz continuous in all variables and satisfy linear growth condition. And then the mean-square stability of such method has been considered.

It should be mentioned that the assumptions of the meansquare convergence in the paper are slightly strong. In the future, we will look for more relaxed conditions to establish new convergence results. In addition, as we mentioned in the introduction section, there are many efficient numerical methods to solve stochastic ordinary differential equations in the existing literature, such as stochastic linear multistep method and stochastic Runge-Kutta methods. Therefore, the adaptation of such methods for stochastic Volterra integrodifferential equations is worth studying in the future.

\section{Conflict of Interests}

The authors declare that there is no conflict of interests regarding the publication of this paper.

\section{Acknowledgments}

The authors are indebted to the referees and the editors for their carefully reading of this paper and their valuable comments. This work was in part supported by NSF of China (nos. 11326240, 11401545, 11371157, and 91130003). The first author was also supported by the Fundamental Research Funds for the Central Universities, China University of Geosciences (Wuhan) (no. CUG120828). The second author was supported by the Fundamental Research Funds for the Central Universities (no. 2013TS137).

\section{References}

[1] T. A. Burton, Volterra Integral and Differential Equations, Elsevier, 2005.

[2] H. Brunner, Collocation Methods for Volterra Integral and Related Functional Differential Equations, Cambridge University Press, 2004.

[3] A. Feldstein and J. R. Sopka, "Numerical methods for nonlinear Volterra integro-differential equations," SIAM Journal on Numerical Analysis, vol. 11, pp. 826-846, 1974.

[4] P. Linz, "Linear multistep methods for Volterra integro-differential equations," Journal of the Association for Computing Machinery, vol. 16, pp. 295-301, 1969.

[5] X. R. Mao, "Stability of stochastic integro differential equations," Stochastic Analysis and Applications, vol. 18, no. 6, pp. 1005-1017, 2000 . 
[6] X. R. Mao and M. Riedle, "Mean square stability of stochastic Volterra integro-differential equations," Systems and Control Letters, vol. 55, no. 6, pp. 459-465, 2006.

[7] J. A. D. Appleby, "pth mean integrability and almost sure asymptotic stability of solutions of It $\hat{o}$-volterra equations," Journal of Integral Equations and Applications, vol. 15, no. 4, pp. 321-341, 2003.

[8] E. Buckwar and R. Winkler, "Multistep methods for SDEs and their application to problems with small noise," SIAM Journal on Numerical Analysis, vol. 44, no. 2, pp. 779-803, 2006.

[9] E. Buckwar and R. Winkler, "Improved linear multi-step methods for stochastic ordinary differential equations," Journal of Computational and Applied Mathematics, vol. 205, no. 2, pp. 912-922, 2007.

[10] K. Burrage, P. M. Burrage, and T. Tian, "Numerical methods for strong solutions of stochastic differential equations: an overview," Proceedings of The Royal Society of London A: Mathematical, Physical and Engineering Sciences, vol. 460, no. 2041, pp. 373-402, 2004.

[11] T. H. Tian and K. Burrage, "Implicit Taylor methods for stiff stochastic differential equations," Applied Numerical Mathematics, vol. 38, no. 1-2, pp. 167-185, 2001.

[12] P. Burrage, Runge-Kutta methods for stochastic differential equations [Ph.D. thesis], University of Queensland, Brisbane, Australia, 1999.

[13] P. E. Kloeden and E. Platen, Numerical Solution of Stochastic Differential Equations, Springer, Berlin, Germany, 1992.

[14] J. Golec and S. Sathananthan, "Sample path approximation for stochastic integro-differential equations," Stochastic Analysis and Applications, vol. 17, no. 4, pp. 579-588, 1999.

[15] J. Golec and S. Sathananthan, "Strong approximations of stochastic integro-differential equations," Dynamics of Continuous, Discrete \& Impulsive Systems B: Applications \& Algorithms, vol. 8, no. 1, pp. 139-151, 2001.

[16] L. E. Shaikhet and J. A. Roberts, "Reliability of difference analogues to preserve stability properties of stochastic Volterra integro-differential equations," Advances in Difference Equations, vol. 2006, Article ID 73897, 22 pages, 2006.

[17] D. J. Higham, "Mean-square and asymptotic stability of the stochastic theta method," SIAM Journal on Numerical Analysis, vol. 38, no. 3, pp. 753-769, 2000.

[18] D. J. Higham, "A-stability and stochastic mean-square stability," BIT Numerical Mathematics, vol. 40, no. 2, pp. 404-409, 2000.

[19] X. Mao, Stochastic Differential Equations and Applications, Horwood, Chichester, UK, 2nd edition, 2008.

[20] C. T. H. Baker and E. Buckwar, "Continuous $\theta$-methods for the stochastic pantograph equation," Electronic Transactions on Numerical Analysis, vol. 11, pp. 131-151, 2000. 


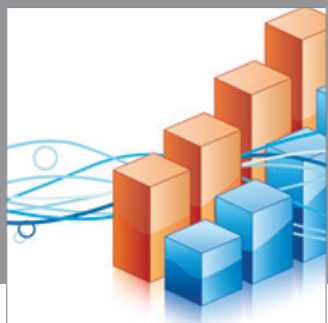

Advances in

Operations Research

mansans

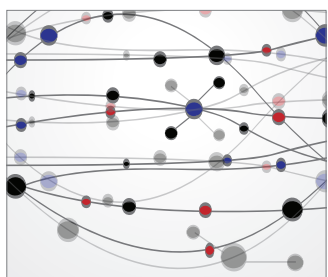

The Scientific World Journal
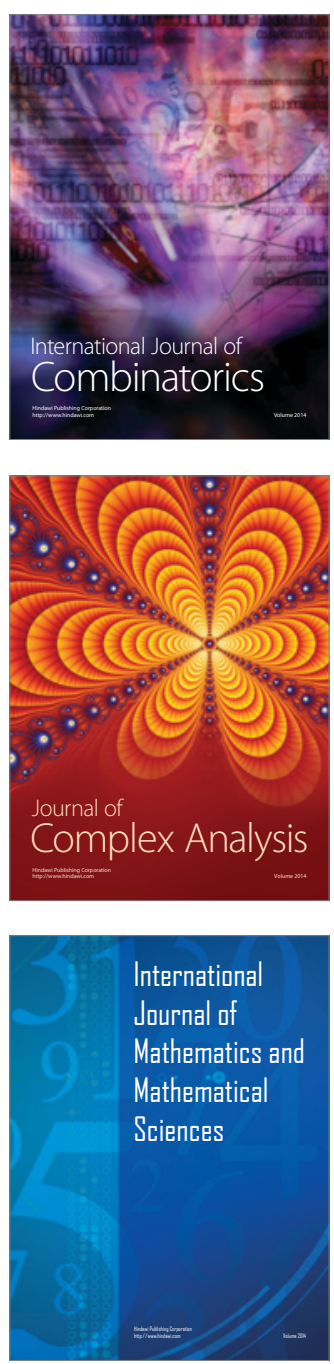
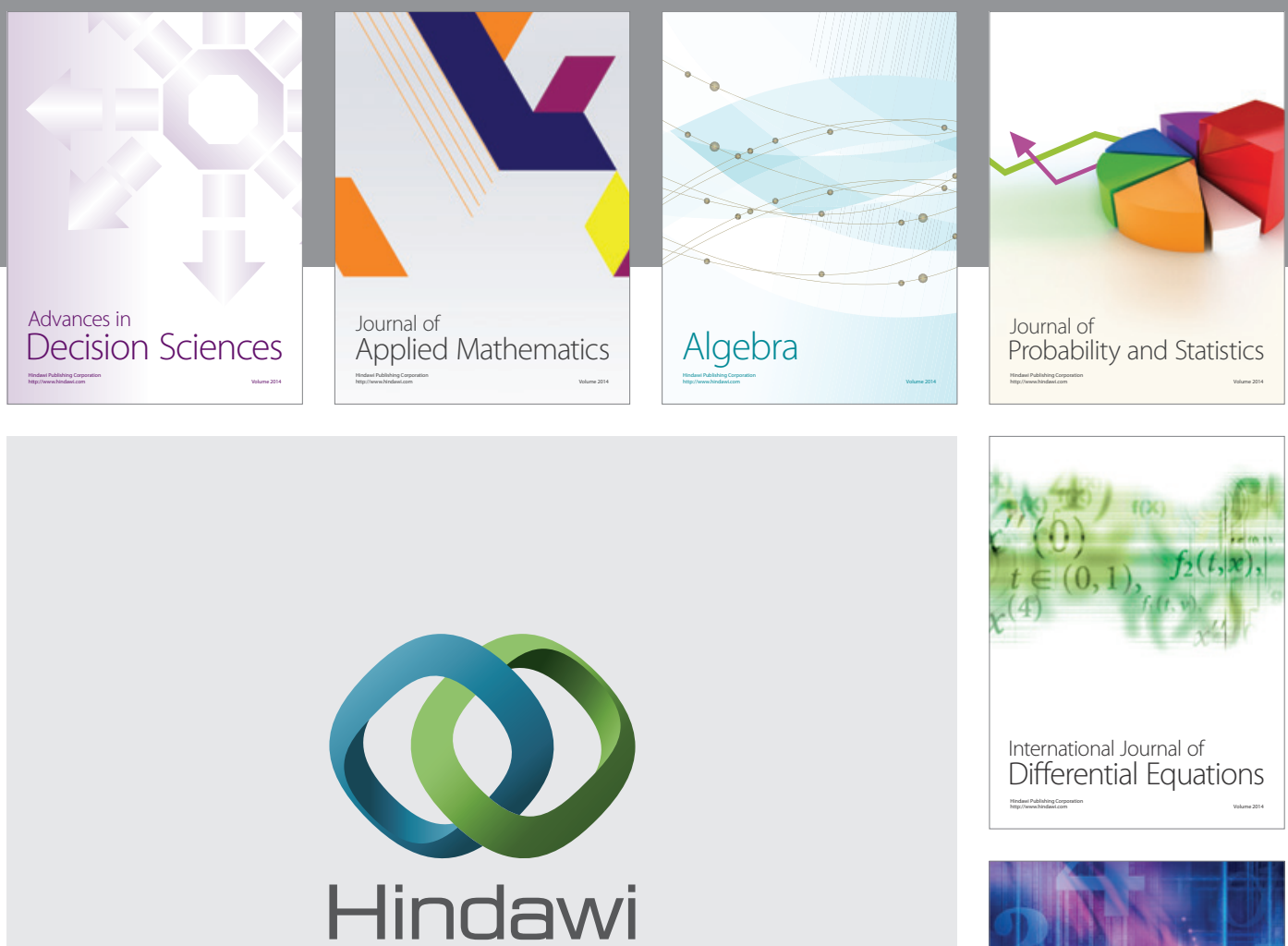

Submit your manuscripts at http://www.hindawi.com
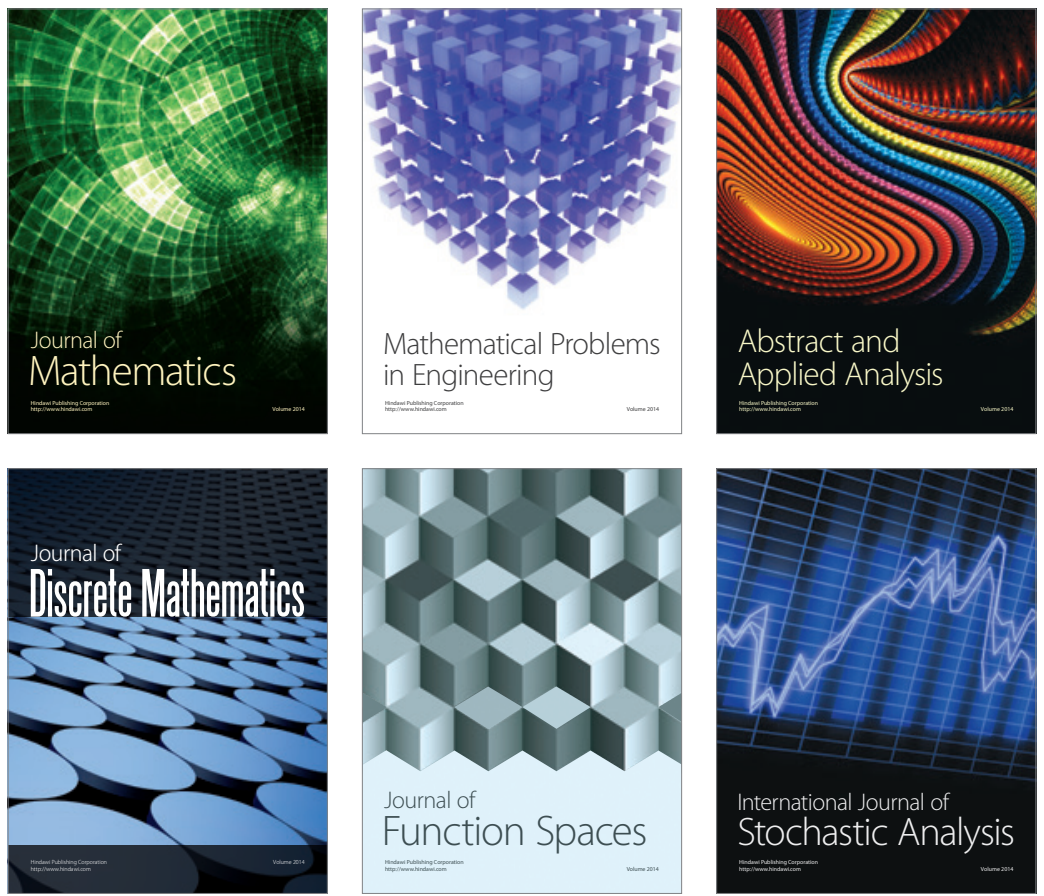

Journal of

Function Spaces

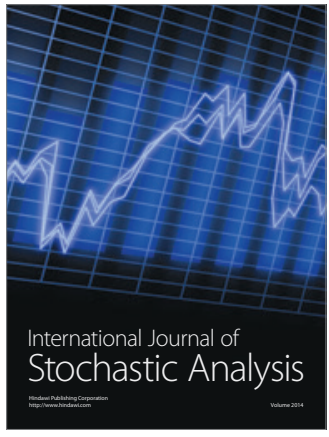

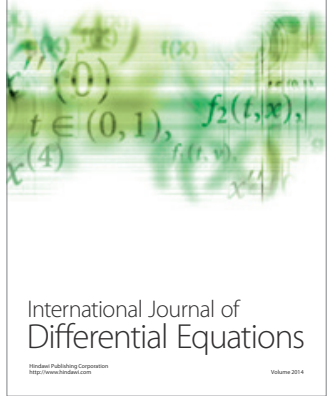
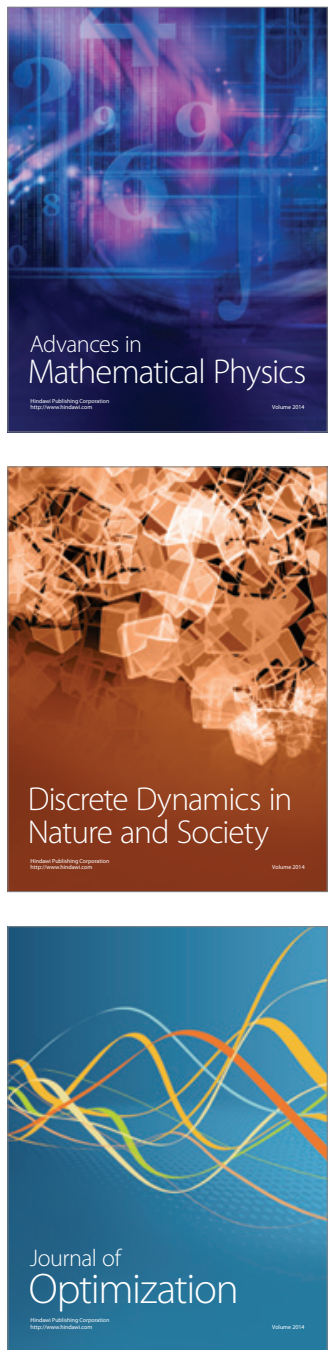